\title{
ESSENTIAL G-RADICAL SUPPLEMENTED MODULES
}

\author{
CELIL NEBIYEV AND HASAN HÜSEYIN ÖKTEN
}

Received 18 January, 2021

\begin{abstract}
Let $M$ be an $R$-module. If every essential submodule of $M$ has a g-radical supplement in $M$, then $M$ is called an essential g-radical supplemented (or briefly eg-radical supplemented) module. In this work, some properties of these modules are investigated. It is proved that every factor module and every homomorphic image of an eg-radical supplemented module are eg-radical supplemented. Let $M$ be an eg-radical supplemented module. Then every finitely $M$-generated $R$-module is eg-radical supplemented.
\end{abstract}

2010 Mathematics Subject Classification: 16D10; 16D70

Keywords: essential submodules, g-small submodules, generalized radical, g-supplemented modules

\section{INTRODUCTION}

Throughout this paper all rings are associative with identity and all modules are unital left modules.

Let $R$ be a ring and $M$ be an $R$-module. We denote a submodule $N$ of $M$ by $N \leq M$. A module $M$ is said to be simple if $M$ have no submodules with distinct from 0 and $M$. The sum of all simple submodules of a module $M$ is called the socle of $M$ and denoted by $\operatorname{Soc}(M) . M$ is called a semisimple module, if $M$ is a direct sum of simple modules (it is equivalent to $\operatorname{Soc}(M)=M$ ). Let $M$ be an $R$-module and $N \leq M$. If $L=M$ for every submodule $L$ of $M$ such that $M=N+L$, then $N$ is called a small (or superfluous) submodule of $M$ and denoted by $N \ll M$. A submodule $N$ of an $R$-module $M$ is called an essential submodule, denoted by $N \unlhd M$, in case $K \cap N \neq 0$ for every submodule $K \neq 0$, or equvalently, $N \cap L=0$ for $L \leq M$ implies that $L=0$. Let $M$ be an $R$-module and $K$ be a submodule of $M . K$ is called a generalized small (briefly $g$-small) submodule of $M$ if for every essential submodule $T$ of $M$ with the property $M=K+T$ implies that $T=M$, we denote this by $K \ll_{g} M$ (in [13], it is called an $e$-small submodule of $M$ and denoted by $K \ll_{e} M$ ). Let $M$ be an $R$-module and $U, V \leq M$. If $M=U+V$ and $V$ is minimal with respect to this property, or equivalently, $M=U+V$ and $U \cap V \ll V$, then $V$ is called a supplement of $U$ in $M . M$ is said to be supplemented if every submodule of $M$ has a supplement in $M . M$ is said to be essential supplemented (briefly e-supplemented) 
if every essential submodule of $M$ has a supplement in $M$. Let $M$ be an $R$-module and $U, V \leq M$. If $M=U+V$ and $M=U+T$ with $T \unlhd V$ implies that $T=V$, or equivalently, $M=U+V$ and $U \cap V \ll_{g} V$, then $V$ is called a $g$-supplement of $U$ in $M$. $M$ is said to be $g$-supplemented if every submodule of $M$ has a $g$-supplement in $M$. $M$ is said to be essential $g$-supplemented if every essential submodule of $M$ has a g-supplement in $M$. The intersection of all maximal submodules of an $R$-module $M$ is called the radical of $M$ and denoted by $\operatorname{Rad}(M)$. If $M$ have no maximal submodules, then we denote $\operatorname{Rad}(M)=M$. Let $M$ be an $R$-module and $U, V \leq M$. If $M=U+V$ and $U \cap V \leq \operatorname{Rad}(V)$, then $V$ is called a generalized (Radical) supplement (briefly Rad-supplement) of $U$ in $M . M$ is said to be generalized (Radical) supplemented (briefly Rad-supplemented) if every submodule of $M$ has a Rad-supplement in $M$. The intersection of all essential maximal submodules of an $R$-module $M$ is called the generalized radical (briefly g-radical) of $M$ and denoted by $\operatorname{Rad}_{g} M$ (in [13] it is denoted by $\operatorname{Rad}_{e} M$ ). If $M$ have no essential maximal submodules, then we denote $\operatorname{Rad}_{g} M=M$. Let $M$ be an $R$-module and $U, V \leq M$. If $M=U+V$ and $U \cap V \leq \operatorname{Rad}_{g} V$, then $V$ is called a $g$-radical supplement of $U$ in $M . M$ is said to be g-radical supplemented if every submodule of $M$ has a g-radical supplement in $M$. Let $M$ be an $R$-module and $K \leq V \leq M$. We say $V$ lies above $K$ in $M$ if $V / K \ll M / K$.

More details about supplemented modules are in $[2,11]$. More details about essential supplemented modules are in $[7,8]$. More informations about $\mathrm{g}$-small submodules and g-supplemented modules are in [3,4]. The definition of essential g-supplemented modules and some properties of them are in [5]. More details about generalized (Radical) supplemented modules are in [10,12]. The definition of g-radical supplemented modules and some properties of them are in [4].

Lemma 1 ([4, Lemma 3]). The following assertions are hold.

(1) If $N \leq M$, then $\operatorname{Rad}_{g} N \leq \operatorname{Rad}_{g} M$.

(2) If $K, L \leq M$, then $\operatorname{Rad}_{g} K+\operatorname{Rad}_{g} L \leq \operatorname{Rad}_{g}(K+L)$.

(3) If $f: M \longrightarrow N$ is an $R$-module homomorphism, then $f\left(\operatorname{Rad}_{g} M\right) \leq \operatorname{Rad}_{g} N$.

\section{ESSENTIAL G-RADICAL SUPPLEMENTED MODULES}

Definition 1 ([6, Definition 1]). Let $M$ be an $R$-module. If every essential submodule of $M$ has a g-radical supplement in $M$, then $M$ is called an essential g-radical supplemented (or briefly eg-radical supplemented) module.

Clearly we can see that every essential g-supplemented module is eg-radical supplemented. But the converse is not true in general (see Example 1 and Example 2). Every g-radical supplemented module is eg-radical supplemented.

Proposition 1. Let $M$ be an eg-radical supplemented $R$-module. If every nonzero submodule of $M$ is essential in $M$, then $M$ is g-radical supplemented.

Proof. Clear from definitions. 
Lemma 2. Let $M$ be an eg-radical supplemented module. Then $M / \operatorname{Rad}_{g} M$ have no proper essential submodules.

Proof. Let $U / \operatorname{Rad}_{g} M \unlhd M / \operatorname{Rad}_{g} M$. Then $U \unlhd M$ and since $M$ is eg-radical supplemented, $U$ has a g-radical supplement $V$ in $M$. Here $M=U+V$ and $U \cap V \leq \operatorname{Rad}_{g} V \leq$ $\operatorname{Rad}_{g} M$. Then $M / \operatorname{Rad}_{g} M=(U+V) / \operatorname{Rad}_{g} M=U / \operatorname{Rad}_{g} M+\left(V+\operatorname{Rad}_{g} M\right) / \operatorname{Rad}_{g} M$ and $U / \operatorname{Rad}_{g} M \cap\left(V+\operatorname{Rad}_{g} M\right) / \operatorname{Rad}_{g} M=\left(U \cap V+\operatorname{Rad}_{g} M\right) / \operatorname{Rad}_{g} M=$ $\operatorname{Rad}_{g} M / \operatorname{Rad}_{g} M=0$. Hence $M / \operatorname{Rad}_{g} M=U / \operatorname{Rad}_{g} M \oplus\left(V+\operatorname{Rad}_{g} M\right) / \operatorname{Rad}_{g} M$ and since $U / \operatorname{Rad}_{g} M \unlhd M / \operatorname{Rad}_{g} M, U / \operatorname{Rad}_{g} M=M / \operatorname{Rad}_{g} M$. Thus $M / \operatorname{Rad}_{g} M$ have no proper essential submodules.

Corollary 1. Let $M$ be an eg-radical supplemented module. Then $M / \operatorname{Rad}_{g} M$ is semisimple.

Proof. Since $M$ is eg-radical supplemented, by Lemma 2, $M / \operatorname{Rad}_{g} M$ have no proper essential submodules. Then by [11, Section 21.1], $\operatorname{Soc}\left(M / \operatorname{Rad}_{g} M\right)=M / \operatorname{Rad}_{g} M$ and $M / \operatorname{Rad}_{g} M$ is semisimple.

Corollary 2. Let $M$ be an essential $g$-supplemented module. Then $M / \operatorname{Rad}_{g} M$ is semisimple.

Proof. Clear from Corollary 1.

Lemma 3. Let $M$ be an $R$-module, $U \unlhd M$ and $N \leq M$. If $U+N$ has a g-radical supplement in $M$ and $N$ is eg-radical supplemented, then $U$ has a g-radical supplement in $M$.

Proof. Let $X$ be a g-radical supplement of $U+N$ in $M$. Since $U \unlhd M, U+X \unlhd$ $M$ and $(U+X) \cap N \unlhd N$. Since $N$ is eg-radical supplemented, $(U+X) \cap N$ has a g-radical supplement $Y$ in $N$. Since $X$ is a g-radical supplement of $U+N$ in $M$, $M=U+N+X$ and $(U+N) \cap X \leq \operatorname{Rad}_{g} X$. Since $Y$ is a g-radical supplement of $(U+X) \cap N$ in $N, N=(U+X) \cap N+Y$ and $(U+X) \cap Y=(U+X) \cap N \cap Y \leq \operatorname{Rad}_{g} Y$. Then $M=U+N+X=U+(U+X) \cap N+Y+X=U+X+Y$ and, by Lemma 1, $U \cap(X+Y) \leq(U+X) \cap Y+(U+Y) \cap X \leq \operatorname{Rad}_{g} Y+(U+N) \cap X \leq \operatorname{Rad}_{g} Y+\operatorname{Rad}_{g} X$ $\leq \operatorname{Rad}_{g}(X+Y)$. Hence $X+Y$ is a g-radical supplement of $U$ in $M$.

Lemma 4. Let $M=M_{1}+M_{2}$. If $M_{1}$ and $M_{2}$ are eg-radical supplemented, then $M$ is also eg-radical supplemented.

Proof. Let $U \unlhd M . \quad$ Then $U+M_{1} \unlhd M$ and since $U+M_{1}+M_{2}$ has a trivial g-radical supplement 0 in $M$ and $M_{2}$ is eg-radical supplemented, by Lemma 3, $U+M_{1}$ has a g-radical supplement in $M$. Since $M_{1}$ is eg-radical supplemented and $U \unlhd M$, by Lemma 3 again, $U$ has a g-radical supplement in $M$. Hence $M$ is eg-radical supplemented.

Corollary 3. Let $M=M_{1}+M_{2}+\cdots+M_{n}$. If $M_{i}$ is eg-radical supplemented for every $i=1,2, \ldots, n$, then $M$ is also eg-radical supplemented. 
Proof. Clear from Lemma 4.

Lemma 5. Let $f: M \rightarrow N$ be an R-module epimorphism, $U, V \leq M$ and $\operatorname{Ker}(f) \leq$ $U$. If $V$ is a g-radical supplement of $U$ in $M$, then $f(V)$ is a g-radical supplement of $f(U)$ in $N$.

Proof. Since $V$ is a g-radical supplement of $U$ in $M, M=U+V$ and $U \cap V \leq$ $\operatorname{Rad}_{g} V$. Then $N=f(M)=f(U+V)=f(U)+f(V)$. Let $x \in f(U) \cap f(V)$. Then there exist $u \in U$ and $v \in V$ with $x=f(u)=f(v)$. Here $f(v-u)=f(v)-f(u)=0$ and $v-u \in \operatorname{Ker}(f) \leq U$. Then $v=v-u+u \in U$ and since $v \in V, v \in U \cap V$. Hence $x=f(v) \in f(U \cap V)$ and $f(U) \cap f(V) \leq f(U \cap V)$. Here clearly we can see that $f(U \cap V) \leq f(U) \cap f(V)$ and $f(U) \cap f(V)=f(U \cap V)$. Since $U \cap V \leq \operatorname{Rad}_{g} V$, by Lemma 1, $f(U) \cap f(V)=f(U \cap V) \leq f\left(\operatorname{Rad}_{g} V\right) \leq \operatorname{Rad}_{g} f(V)$. Hence $f(V)$ is a g-radical supplement of $f(U)$ in $N$, as desired.

Lemma 6. Every homomorphic image of an eg-radical supplemented module is eg-radical supplemented.

Proof. Let $M$ be an eg-radical supplemented $R$-module and $f: M \rightarrow N$ be an $R$ module epimorphism. Let $U \unlhd N$. By [11, Section 17.3 (3)], $f^{-1}(U) \unlhd M$ and since $M$ is eg-radical supplemented, $f^{-1}(U)$ has a g-radical supplement $V$ in $M$. Since $\operatorname{ker}(f) \leq f^{-1}(U)$, by Lemma 5, $f(V)$ is a g-radical supplement of $f\left(f^{-1}(U)\right)=U$ in $N$. Hence $N$ is eg-radical supplemented, as desired.

Corollary 4. Every factor module of an eg-radical supplemented module is egradical supplemented.

Proof. Clear from Lemma 6.

Lemma 7. Let $M$ be an eg-radical supplemented $R$-module. Then every finitely $M$-generated $R$-module is eg-radical supplemented.

Proof. Let $N$ be a finitely $M$-generated $R$-module. Then there exist a finite index set $\Lambda$ and an $R$-module epimorphism $f: M^{(\Lambda)} \rightarrow N$. Since $M$ is eg-radical supplemented, by Corollary $3, M^{(\Lambda)}$ is eg-radical supplemented. Then by Lemma $6, N$ is eg-radical supplemented, as desired.

Proposition 2. Let $R$ be a ring. Then the $R$-module $R$ is eg-radical supplemented if and only if every finitely generated $R$-module is eg-radical supplemented.

Proof. ( $\Longrightarrow$ ) Clear from Lemma 7.

$(\Longleftarrow)$ Clear, since ${ }_{R} R$ is finitely generated.

Definition 2. Let $M$ be an $R$-module and $X \leq M$. If $X$ is a g-radical supplement of an essential submodule of $M$, then $X$ is called an eg-radical supplement submodule in $M$. 
Let $M$ be an $R$-module. It is defined the relation $\beta^{*}$ on the set of submodules of an $R$-module $M$ by $X \beta^{*} Y$ if and only if $Y+K=M$ for every $K \leq M$ such that $X+K=M$ and $X+T=M$ for every $T \leq M$ such that $Y+T=M$ (see [1]). It is defined the relation $\beta_{g}^{*}$ on the set of submodules of an $R$-module $M$ by $X \beta_{g}^{*} Y$ if and only if $Y+K=M$ for every $K \unlhd M$ such that $X+K=M$ and $X+T=M$ for every $T \unlhd M$ such that $Y+T=M$ (see [9]).

Lemma 8. Let $M$ be an R-module. If every essential submodule of $M$ is $\beta_{g}^{*}$ equivalent to an eg-radical supplement submodule in $M$, then $M$ is eg-radical supplemented.

Proof. Let $X \unlhd M$. By hypothesis, there exists an eg-radical supplement submodule $V$ in $M$ with $X \beta_{g}^{*} V$. Let $V$ be a g-radical supplement of an essential submodule $U$ in $M$. Then $M=U+V$ and $U \cap V \leq \operatorname{Rad}_{g} V$. Since $U \unlhd M$, by hypothesis, there exists an eg-radical supplement submodule $Y$ in $M$ with $U \beta_{g}^{*} Y$. Let $Y$ be a g-radical supplement of $S$ in $M$ and $S \unlhd M$. Then $M=S+Y$ and $S \cap Y \leq \operatorname{Rad}_{g} Y$. Since $X \beta_{g}^{*} V$ and $M=U+V, M=X+U$ and since $U \beta_{g}^{*} Y$ and $X \unlhd M, M=X+Y$. Assume $X \cap Y \not \leq \operatorname{Rad}_{g} Y$. Then there exists an essential maximal submodule $T$ of $Y$ such that $X \cap Y+T=Y$. By using [2, Lemma 1.24], we can see that $M=S+Y=X \cap Y+S+T=Y+X \cap(S+T)=U+X \cap(S+T)$ $=X+U \cap(S+T)=V+U \cap(S+T)=U \cap V+S+T$. Since $T$ is an essential maximal submodule of $Y$, by $\frac{M}{S+T}=\frac{Y+S+T}{S+T} \cong \frac{Y}{Y \cap(S+T)}=\frac{Y}{S \cap Y+T}=\frac{Y}{T}$ and $S+T \unlhd M$, $S+T$ is an essential maximal submodule of $M$ and hence $U \cap V \leq \operatorname{Rad}_{g} V \leq S+T$. Then $M=U \cap V+S+T=S+T$. This is a contradiction. Hence $X \cap Y \leq \operatorname{Rad}_{g} Y$ and $Y$ is a g-radical supplement of $X$ in $M$. Thus $M$ is eg-radical supplemented.

Corollary 5. Let $M$ be an R-module. If every essential submodule of $M$ is $\beta^{*}$ equivalent to an eg-radical supplement submodule in $M$, then $M$ is eg-radical supplemented.

Proof. Clear from Lemma 8.

Corollary 6. Let $M$ be an R-module. If every essential submodule of $M$ lies above an eg-radical supplement submodule in $M$, then $M$ is eg-radical supplemented.

Proof. Clear from Corollary 5.

Corollary 7. Let $M$ be an R-module. If every essential submodule of $M$ is an eg-radical supplement submodule in $M$, then $M$ is eg-radical supplemented.

Proof. Clear from Corollary 6.

Lemma 9. Let $M$ be an $R$-module. If every submodule of $M$ is $\beta^{*}$ equivalent to an eg-radical supplement submodule in $M$, then $M$ is g-radical supplemented.

Proof. Let $X \leq M$. By hypothesis, there exists an eg-radical supplement submodule $V$ in $M$ with $X \beta^{*} V$. Let $V$ be a g-radical supplement of an essential submodule $U$ 
in $M$. Then $M=U+V$ and $U \cap V \leq \operatorname{Rad}_{g} V$. By hypothesis, there exists an eg-radical supplement submodule $Y$ in $M$ with $U \beta^{*} Y$. Let $Y$ be a g-radical supplement of an essential submodule $S$ in $M$. Then $M=S+Y$ and $S \cap Y \leq \operatorname{Rad}_{g} Y$. Since $X \beta^{*} V$ and $M=U+V, M=X+U$ and since $U \beta^{*} Y, M=X+Y$. Assume $X \cap Y \not \leq \operatorname{Rad}_{g} Y$. Then there exists an essential maximal submodule $T$ of $Y$ such that $X \cap Y+T=Y$. By using [2, Lemma 1.24], we can see that $M=S+Y=X \cap Y+S+T=Y+X \cap(S+T)=$ $U+X \cap(S+T)=X+U \cap(S+T)=V+U \cap(S+T)=U \cap V+S+T$. Since $T$ is an essential maximal submodule of $Y$, by $\frac{M}{S+T}=\frac{Y+S+T}{S+T} \cong \frac{Y}{Y \cap(S+T)}=\frac{Y}{S \cap Y+T}=\frac{Y}{T}$ and $S+T \unlhd M, S+T$ is an essential maximal submodule of $M$ and hence $U \cap V \leq$ $\operatorname{Rad}_{g} V \leq S+T$. Then $M=U \cap V+S+T=S+T$. This is a contradiction. Hence $X \cap Y \leq \operatorname{Rad}_{g} Y$ and $Y$ is a g-radical supplement of $X$ in $M$. Thus $M$ is g-radical supplemented.

Corollary 8. Let $M$ be an R-module. If every submodule of $M$ lies above an egradical supplement submodule in $M$, then $M$ is g-radical supplemented.

Proof. Clear from Lemma 9.

Corollary 9. Let $M$ be an $R$-module. If every submodule of $M$ is an eg-radical supplement submodule in $M$, then $M$ is g-radical supplemented.

Proof. Clear from Lemma 9.

Example 1. Consider the $\mathbb{Z}$-module $\mathbb{Q}$. Since $\operatorname{Rad}_{g} \mathbb{Q}=\operatorname{Rad}(\mathbb{Q})=\mathbb{Q}, \mathbb{Z} \mathbb{Q}$ is egradical supplemented. But, since $\mathbb{Z} \mathbb{Q}$ is not supplemented and every nonzero submodule of $\mathbb{Z} \mathbb{Q}$ is essential in $\mathbb{Z} \mathbb{Q}, \mathbb{Q}$ is not essential g-supplemented.

Example 2. Consider the $\mathbb{Z}$-module $\mathbb{Q} \oplus \mathbb{Z}_{p^{2}}$ for a prime $p$. It is easy to check that $\operatorname{Rad}_{g} \mathbb{Z}_{p^{2}} \neq \mathbb{Z}_{p^{2}}$. By [4, Lemma 4], $\operatorname{Rad}_{g}\left(\mathbb{Q} \oplus \mathbb{Z}_{p^{2}}\right)=\operatorname{Rad}_{g} \mathbb{Q} \oplus \operatorname{Rad}_{g} \mathbb{Z}_{p^{2}} \neq \mathbb{Q} \oplus \mathbb{Z}_{p^{2}}$. Since $\mathbb{Q}$ and $\mathbb{Z}_{p^{2}}$ are eg-radical supplemented, by Lemma $4, \mathbb{Q} \oplus \mathbb{Z}_{p^{2}}$ is eg-radical supplemented. But $\mathbb{Q} \oplus \mathbb{Z}_{p^{2}}$ is not essential g-supplemented.

\section{REFERENCES}

[1] G. F. Birkenmeier, F. T. Mutlu, C. Nebiyev, N. Sokmez, and A. Tercan, "Goldie*supplemented modules," Glasgow Mathematical Journal, vol. 52A, pp. 41-52, 2010, doi: 10.1017/S0017089510000212.

[2] J. Clark, C. Lomp, N. Vanaja, and R. Wisbauer, Lifting modules: supplements and projectivity in module theory (frontiers in mathematics), 2006th ed. Basel: Birkhäuser, 82006. doi: 10.1007/3-7643-7573-6.

[3] B. Koşar, C. Nebiyev, and N. Sökmez, "g-supplemented modules," Ukrainian Mathematical Journal, vol. 67, no. 6, pp. 861-864, 2015, doi: 10.1007/s11253-015-1127-8.

[4] B. Koşar, C. Nebiyev, and A. Pekin, "A generalization of g-supplemented modules," Miskolc Math. Notes, vol. 20, no. 1, pp. 345-352, 2019, doi: 10.18514/MMN.2019.2586.

[5] C. Nebiyev and H. H. Ökten, "Essential g-supplemented modules," Turkish Studies Information Technologies and Applied Sciences, vol. 14, no. 1, pp. 83-89, 2019.

[6] C. Nebiyev and H. H. Ökten, "eg-radical supplemented modules," in 3rd International EConference on Mathematical Advances and Applications (ICOMAA-2020), 2020. 
[7] C. Nebiyev, H. H. Ökten, and A. Pekin, "Amply essential supplemented modules," Journal of Scientific Research and Reports, vol. 24, no. 4, pp. 1-4, 2018, doi: 10.9734/JSRR/2018/45651.

[8] C. Nebiyev, H. H. Ökten, and A. Pekin, "Essential supplemented modules," International Journal of Pure and Applied Mathematics, vol. 120, no. 2, pp. 253-257, 2018, doi: 10.12732/ijpam.v120i2.9.

[9] C. Nebiyev and N. Sökmez, "Beta g-star relation on modules," Eur. J. Pure Appl. Math., vol. 11, no. 1, pp. 238-243, 2018, doi: 10.29020/nybg.ejpam.v11i1.2741.

[10] Y. Wang and N. Ding, "Generalized supplemented modules," Taiwanese Journal of Mathematics, vol. 10, no. 6, pp. 1589-1601, 2006, doi: 10.11650/twjm/1500404577.

[11] R. Wisbauer, Foundations of module and ring theory, german ed., ser. Algebra, Logic and Applications. Gordon and Breach Science Publishers, Philadelphia, PA, 1991, vol. 3, a handbook for study and research, doi: 10.1201/9780203755532.

[12] W. Xue, "Characterizations of semiperfect and perfect rings," Publicacions Matematiques, vol. 40, no. 1, pp. 115-125, 1996.

[13] D. X. Zhou and X. R. Zhang, "Small-essential submodules and morita duality," Southeast Asian Bulletin of Mathematics, vol. 35, pp. 1051-1062, 2011.

Authors' addresses

Celil Nebiyev

(Corresponding author) Ondokuz Mayıs University, Department of Mathematics, Atakum, Samsun, Turkey

E-mail address: cnebi yev@omu.edu.tr

\section{Hasan Hüseyin Ökten}

Amasya University, Technical Sciences Vocational School, Amasya, Turkey

E-mail address: hokten@gmail.com 